\title{
Influence of the Sample Volume upon Injection on Capillary Gel Electrophoresis for the Separation of Small DNA Fragments
}

\author{
Tomoka Nakazumi and Yusuke Hara
}

\begin{abstract}
In this study, we evaluated the influence of the sample volume (10 $\mu \mathrm{L}$ and $20 \mu \mathrm{L})$ upon the injection to a developed capillary gel electrophoresis (CGE) system designed using a short-length capillary (total length of $15 \mathrm{~cm}$ and effective length of $7.5 \mathrm{~cm}$ ). The fused silica capillary was coated with an acrylamide linear polymer chain to prevent the electroosmotic flow in the direction opposite to the movement of DNA samples. The sieving polymer solution used was hydroxyethyl cellulose with a molecular weight of $1,300,000$. Using this CGE system for the separation of a 100-bp DNA Ladder sample, the sample injection volume had a substantial effect on the electropherogram results. The error bars for migration time, mobility, and resolution length were much larger when using 20 $\mu \mathrm{L}$ of the sample than using $10 \mu \mathrm{L}$. The use of $10 \mu \mathrm{L}$ of the sample provided highly reliable results. These findings indicated that developers of CGE equipment should pay close attention to the sample injection volume in order to measure small DNA samples with high accuracy.
\end{abstract}

Index Terms-Capillary gel electrophoresis, injection, point-of-care testing, polymer solution.

\section{INTRODUCTION}

Capillary gel electrophoresis (CGE) is recognized as a very useful tool for the separation of products after polymerase chain reaction (PCR), with greater reliability compared to slab gel electrophoresis (SGE) [1]-[4], owing to its speed, requirement of a very small amount of DNA, and higher accuracy. Recently, there has been great worldwide interest for an immediate compact analysis tool in point-of-care testing (POCT) to detect diseases and bacteria that can be used in bedside applications such in hospitals. To meet this demand, we are challenging development of a compact CGE system for the analysis of PCR products. To apply the CGE method to a compact system for POCT, the length of the fused silica capillary needs to be shortened while maintaining a high accuracy and resolution. To separate a DNA sample using the fused silica capillary, the capillary should first be coated because the capillary walls cause electroosmotic flow (EOF) in an opposite direction to the flow of the DNA samples due to the negative charge. Indeed, when using an untreated capillary, the DNA samples cannot be inserted into the capillary because of the inhibition by the EOF. Therefore, a coating method for

Manuscript received December 4, 2017; revised February 5, 2018.

Tomoka Nakazumi and Yusuke Hara were with Research Institute for Sustainable Chemistry, ISC, National Institute of Advanced Industrial Science and Technology, AIST, Central 5-2, 1-1-1 Higashi, Tsukuba 305-8565, Japan (e-mail: y-hara@ aist.go.jp). a fused silica capillary has been extensively investigated [5]-[10]. In this study, to prevent the EOF during the process of introducing the DNA samples, a non-ionic acrylamide polymer chain was adopted via chemical bonding to the fused silica wall. In addition, a sieving polymer is needed to separate small DNA samples [11]-[13]. Since the DNA samples and the sieving polymer chain entangle, the migration time of the DNA with different lengths are different [14], [15]. To separate DNA samples clearly, we adopted hydroxyethyl cellulose (HEC) with a molecular weight of 1,300,000.

In our experiments, to separate a 100-bp DNA Ladder sample accurately with the developed CGE system, we evaluated the influence of the DNA samples volume upon the injection on the electropherogram results based on comparisons of the migration time, mobility, and resolution length.

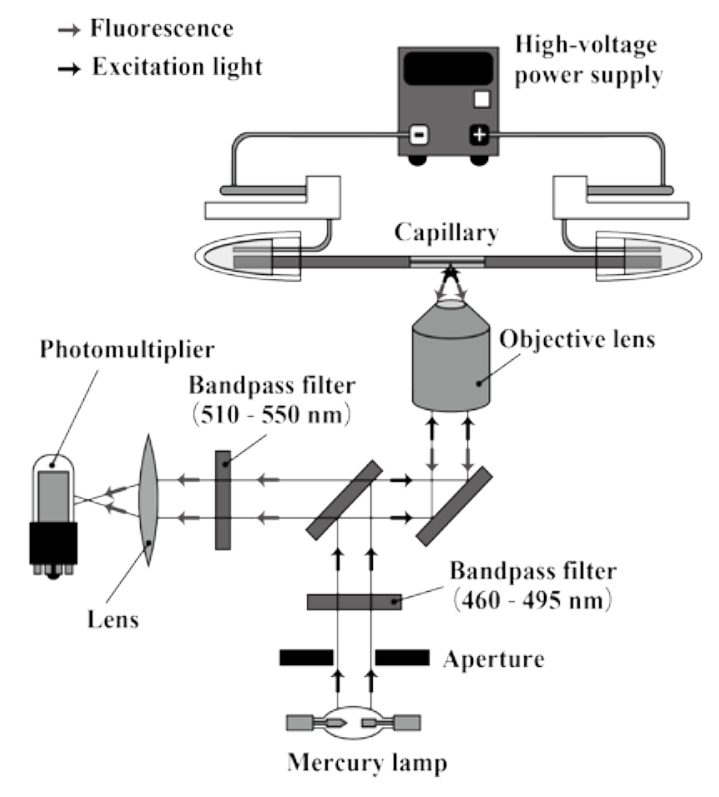

Fig. 1. Schematic illustration of the capillary gel electrophoresis system.

\section{EXPERIMENTAL SECTION}

\section{A. CGE Measurement System}

The CGE system (Fig. 1) consisted of a microscope with epi-illumination (IX73; Olympus, Tokyo, Japan) and a high-voltage power supply (HJPQ-10P3; Matsusada, Osaka, Japan). The excitation light source was a mercury lamp using an optical filter (U-FBWA; Olympus, Tokyo, Japan). For this study, SYBR Green II, which conjugates to the DNA samples, 
was used as the fluorescent chemical compound. The fluorescence from the DNA samples was captured using a 60× objective lens (UPlanFLN; Olympus), and the fluorescence signal was detected using a photomultiplier tube (H8249-101; Hamamatsu Photonics, Hamamatsu, Japan). The signal was converted to digital data for quantification with the National Instrument NI USB-6341. The diameter of the fused silica capillary (Polymicro Technologies, Phoenix, AZ, USA) was $75 \mu \mathrm{m}$, with a total length of $15 \mathrm{~cm}$ and an effective length of $7.5 \mathrm{~cm}$. The DNA samples were injected into the capillary at a voltage of $1.5 \mathrm{kV}$ for $1 \mathrm{~s}$, and the separation process was performed at $100 \mathrm{~V} / \mathrm{cm}$.
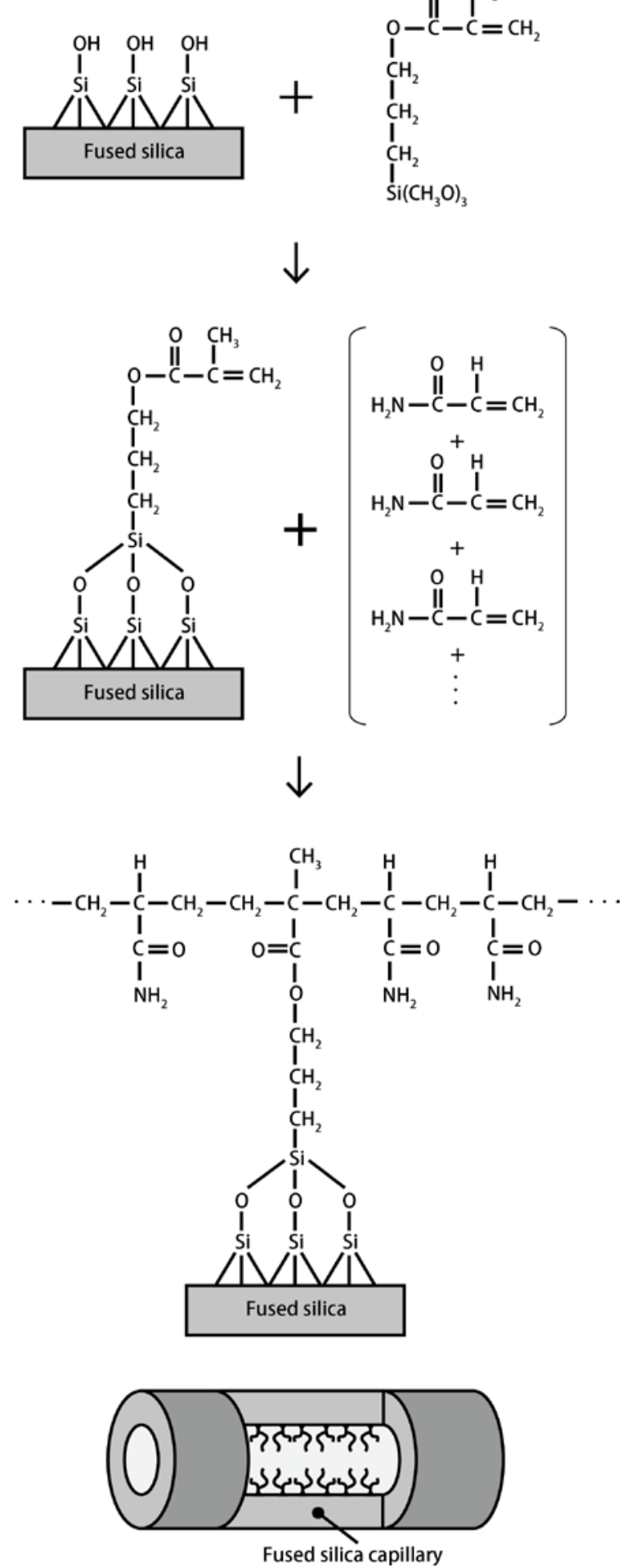

Fig. 2. Schematic illustration of the polymer coating method.

\section{B. Coating Method for the Fused Silica Capillary and CGE Chemicals}

An overview of the overall process of the coating method for the fused silica capillary was shown in Fig. 2. Firstly, the capillary was washed using $1 \mathrm{~N} \mathrm{NaOH}$ (15 min), water (15 min), and methanol (15 min). Then, a solution (total $20 \mathrm{~mL}$ ) containing $80 \mu \mathrm{L}$ of 3-methacryloxypropyltrimethoxysilane (Shin-Etsu Chemical, Tokyo, Japan), $1 \mathrm{~mL}$ of methanol, and one drop of acetic acid was flowed into the capillary to coat the capillary walls. In the next step, the monomer solution (total $20 \mathrm{~mL}$ ), including acrylamide monomer $(0.7 \mathrm{~g})$, ammonium persulfate (20 mg), and $N, N, N^{\prime}, N^{\prime}$-tetramethylethylenediamine $(20 \mu \mathrm{L})$, was flowed into the capillary for $2 \mathrm{~h}$. Before flowing the monomer solution, the solution was bubbled with nitrogen gas for 30 min. The 100-bp DNA Ladder (TAKARA BIO, Shiga, Japan) consisting of 11 double-stranded fragments with lengths of 100, 200, 300, 400, 500, 600, 700, 800, 900, 1000, and 1500 bp was selected as the separation target. The DNA ladder sample $(130 \mu \mathrm{g} / \mathrm{mL})$ was used after it was diluted 10 times with ultrapure water. For the present evaluation, the DNA ladder sample solutions were prepared at two volumes $(10 \mu \mathrm{L}$ and $20 \mu \mathrm{L})$ with the same DNA concentration $(13 \mu \mathrm{g} / \mathrm{mL})$ as the injection samples. A $0.5 \times$ Tris-borate-ethylenediaminetetraacetic acid buffer was selected as the running buffer.

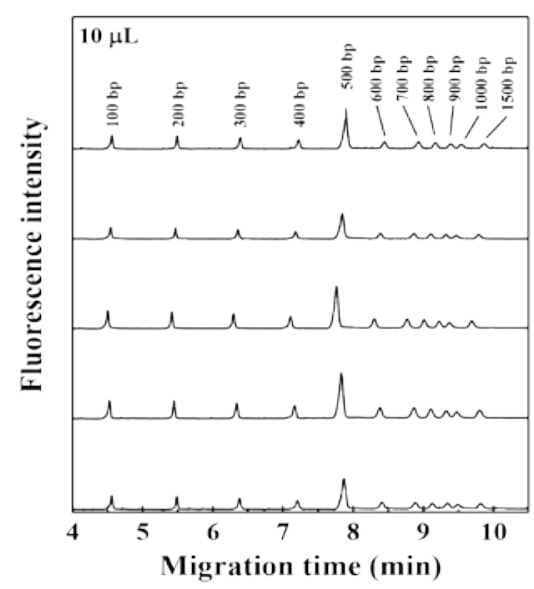

(A)

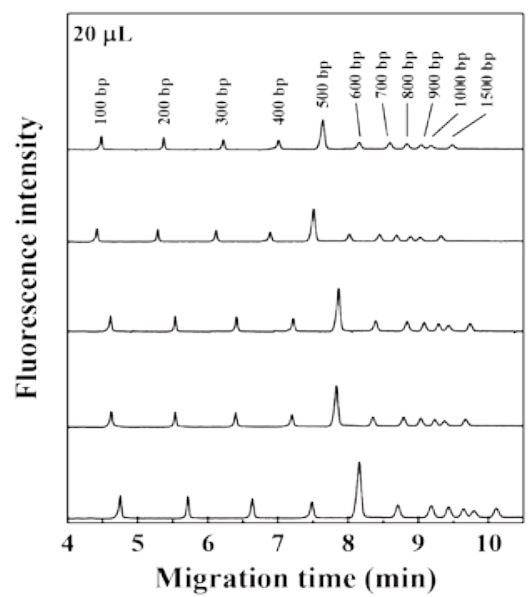

(B)

Fig. 3. Separation of the 100-bp DNA Ladder sample using different DNA sample volumes upon injection: (A) $10 \mu \mathrm{L}$ and (B) $20 \mu \mathrm{L}$. 


\section{RESULTS AND DisCUSSION}

Fig. 3 shows the results of the separation of 100 bp DNA Ladder samples with two different volumes $(10 \mu \mathrm{L}$ and $20 \mu \mathrm{L})$ upon the injection. The electropherograms showed that the experimental condition was suitable for clearly separating the 100-bp DNA Ladder sample. However, the migration time differed depending on the sample volume upon injection. In particular, when $20 \mu \mathrm{L}$ of the sample was used, the migration time varied among CGE measurements. As shown in Fig. 4, the error bars were relatively large at all points for $20 \mu \mathrm{L}$, whereas the error bars were small for $10 \mu \mathrm{L}$ of the sample (Fig. $4 \mathrm{~A}$ vs. Fig. 4B). This trend indicated that the sample volume upon injection substantially affected the injection of DNA samples when using the short-length capillary in this CGE process. The main cause of this phenomenon is that the DNA position in the capillary was not stable after insertion since the applied state of the voltage at the injection is affected by the sample volume. Similarly, the error bars for mobility were higher for $20 \mu \mathrm{L}$ than for $10 \mu \mathrm{L}$ (Fig. 5).

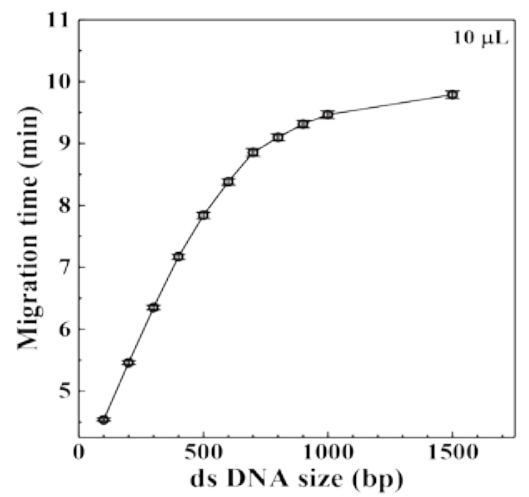

(A)

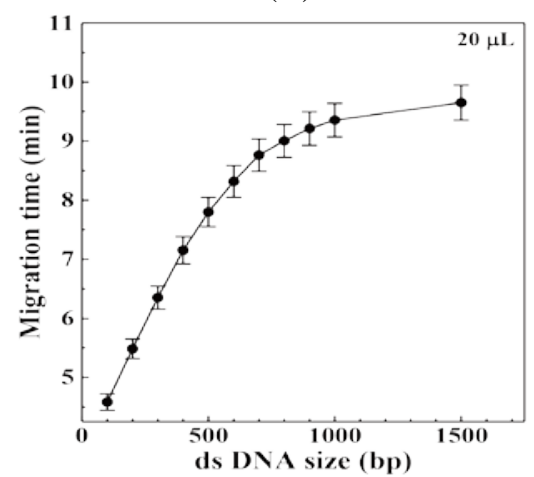

(B)

Fig. 4. Relationship between migration time and dsDNA size (bp) using different DNA sample volumes upon injection: (A) $10 \mu \mathrm{L}$ and (B) $20 \mu \mathrm{L}$.

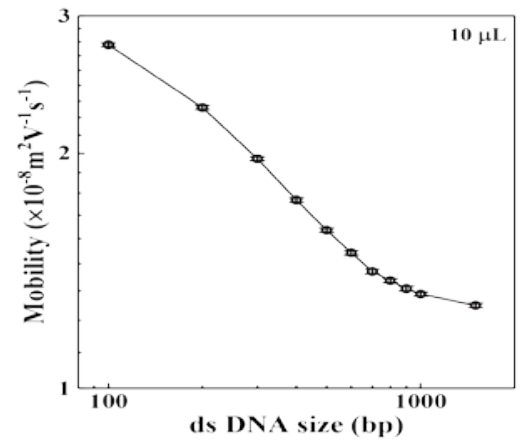

(A)

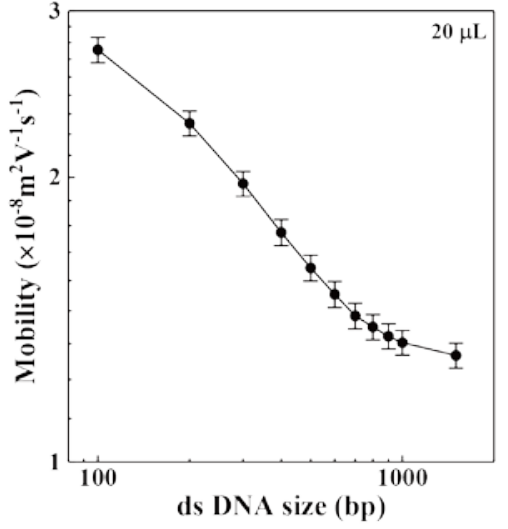

(B)

Fig. 5. Relationship between mobility and dsDNA size (bp) using different DNA sample volumes upon injection: (A) $10 \mu \mathrm{L}$ and (B) $20 \mu \mathrm{L}$.

In addition, Fig. 6 shows the relationship between the resolution length (RSL) and DNA size. The resolution (Rs) was calculated according to the following equation:

$$
R s=1.18 \times \Delta t /\left(w_{0.5}(1)+w_{0.5}(2)\right)
$$

where $\Delta t$ is the difference in the migration time between two consecutive peaks in the electropherograms, and $w_{0.5}$ is the full width at half maximum of the peak in the graph. The RSL was determined using the following equation:

$$
\mathrm{RSL}=\Delta n / R s,
$$

where $\Delta n$ is the DNA length difference for adjacent peaks in the graph, and $R s$ is the resolution value.

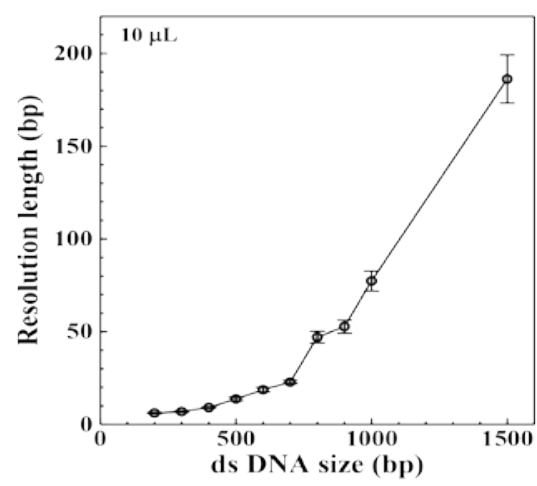

(A)

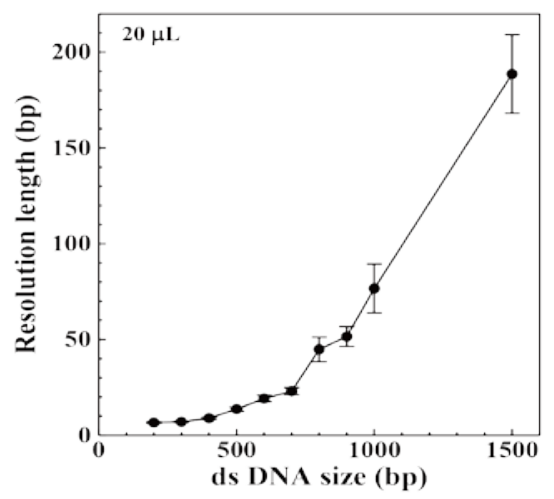

(B)

Fig. 6. Relationship between resolution length (bp) and dsDNA size (bp) using different DNA sample volumes upon injection: (A) $10 \mu \mathrm{L}$ and (B) $20 \mu \mathrm{L}$ 
As shown in Fig. 6, there was much greater variation in the RSL when using $20 \mu \mathrm{L}$ of sample than using $10 \mu \mathrm{L}$ of the sample. This result indicated that the experimental condition of $20 \mu \mathrm{L}$ did not allow for a stable DNA flow rate, whereas flow stability was maintained using $10 \mu \mathrm{L}$ as the injection volume. We considered that this difference is likely caused by the position and spread of the DNA in the capillary after insertion. Based on these results, we concluded that developers of CGE systems need to pay close attention to setting the appropriate sample amount for injection in order to accurately measure small DNA fragments.

\section{CONCLUSION}

In this study, we measured the effect of 100-bp DNA Ladder sample volume $(10 \mu \mathrm{L}$ and $20 \mu \mathrm{L})$ on the CGE process using a developed CGE system. To measure small DNA samples, a short-length capillary (total length of $15 \mathrm{~cm}$ and effective length of $7.5 \mathrm{~cm}$ ) coated with a non-ionic acrylamide polymer chain was adopted to prevent inhibition from the EOF. HEC with a molecular size of 1,300,000 was adopted as the sieving polymer solution. Measurement of the CGE at different volumes $(10 \mu \mathrm{L}$ and $20 \mu \mathrm{L})$ upon the injection showed that the sample volume had a substantial effect on the electropherogram results. Variation in migration time, mobility, and RSL were much greater for the larger sample volume than for the smaller volume. These results indicated that the DNA sample volume upon injection has a large influence on the position and spread of DNA in the capillary. This observation indicated that the applied voltage state during injection differs depending on the sample volume. Thus, a developer of a compact CGE system should pay close attention to setting the sample volume upon the injection so as to ensure separating DNA samples with accuracy.

\section{ACKNOWLEDGMENT}

This work was supported by Grants-in-Aid (KAKENHI) for Young Scientists (B) (16K17493, 15K18014) and Scientific Research (B) (15H03827).

\section{REFERENCES}

[1] P. G. Righetti, Capillary Electrophoresis in Analytical Biotechnology, Boca Raton, FL: CRC Press, 1996.

[2] K. R. Mitchelson and J. Cheng, "Capillary electrophoresis of nucleic acids volume ii: Practical application of capillary electrophoresis," in Methods in Molecular Biology, Springer, 2001, vol. 163.

[3] Paulus, E. Gassmann, and M. J. Field, “Calibration of polyacrylamide gel columns for the separation of oligonucleotides by capillary electrophoresis," Electrophoresis, vol. 11, p. 702, 1990.
[4] A. Guttman, "Gel and polymer-solution mediated separation of biopolymers by capillary electrophoresis," Journal of Chromatographic Science, vol. 41, p. 449, 2003.

[5] D. N. Heiger, A. S. Cohen, and B. L. Karger, "Separation of DNA restriction fragments by high performance capillary electrophoresis with low and zero crosslinked polyacrylamide using continuous and pulsed electric fields," Journal of Chromatography, vol. 516, p. 33, 1990.

[6] T. Manabe, N. Chen, S. Terabe, M. Yohda, and I. Endo, "Effects of linear polyacrylamide concentrations and applied voltages on the separation of oligonucleotides and DNA sequencing fragments by capillary electrophoresis," Analytical Chemistry, vol. 66, p. 4243, 1994.

[7] A. S. Cohen, D. R. Najarian, A. Paulus, A. Guttman, J. A. Smith, and B. L. Karger, "Rapid separation and purification of oligonucleotides by high-performance capillary gel electrophoresis,” vol. 85, p. 9660, 1988.

[8] W. Wei and E. S. Yeung, "Improvements in DNA sequencing by capillary electrophoresis at elevated temperature using poly (ethylene oxide) as a sieving matrix," Journal of Chromatography B, vol. 745, p. 221, 2000.

[9] V. Dolnik and W. A. Gurske, "Capillary electrophoresis in sieving matrices: selectivity per base, mobility slope, and inflection slope," Electrophoresis, vol. 20, p. 3373, 1999.

[10] C. Barta, Z. Ronai, M. Sasvari-Szekely, and A. Guttman, "Rapid single nucleotide polymorphism analysis by primer extension and capillary electrophoresis using polyvinyl pyrrolidine matrix," Electrophoresis, vol. 22, p. 779, 2001.

[11] J. Horvath and V. Dolník, "Polymer wall coatings for capillary electrophoresis,” Electrophoresis, vol. 22, p. 644, 2001.

[12] C.-Y. Liu, "Stationary phases for capillary electrophoresis and capillary electrochromatography,” Electrophoresis, vol. 22, p. 612, 2001.

[13] A. Cifuentes, P. Canalejas, and J. C. Diez-Masa, "Preparation of linear polyacrylamide-coated capillaries: Study of the polymerization process and its effect on capillary electrophoresis performance," Journal of Chromatography A, vol. 830, p. 423, 1999.

[14] Y. Masubuchi, H. Oana, M. Matsumoto, M. Doi, and K. Yoshikawa, "Conformational dynamics of DNA during biased sinusoidal field gel electrophoresis,” Electrophoresis, vol. 17, p. 1065, 1996.

[15] H. Oana, Y. Masubuchi, M. Matsumoto, M. Doi, Y. Matsuzawa, and K. Yoshikawa, "Periodic motion of large DNA molecules during steady field gel electrophoresis,” Macromolecules, vol. 27, p. 6061, 1994.

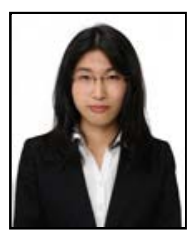

Tomoka Nakazumi received the B.S. degree from Hokkaido University, Hokkaido, Japan, in 2009, and the M.S. and Dr. Sci. degrees from the Tokyo Institute of Technology, Tokyo, Japan, in 2014. She is currently a researcher with Clever-Material Engineering Group, Research Institute for Sustainable Chemistry (ISC), National Institute of Advanced Industrial Science and Technology (AIST), Tsukuba, Japan. Her current research interests include photochemistry, polymer science, and soft actuators. Dr. Nakazumi is a member of the Chemical Society of Japan.

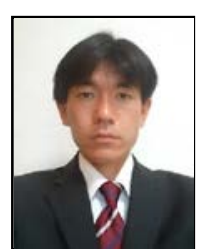

Yusuke Hara received the M.S. degree in the arts from Nagoya University, Nagoya, Japan, in 2001, and the Dr. Eng. degree in materials engineering from the Department of Materials Engineering, Graduate School of Engineering, University of Tokyo, Tokyo, Japan, in 2007. He is currently a senior researcher with Clever-Material Engineering Group, Research Institute for Sustainable Chemistry (ISC), National Institute of Advanced Industrial Science and Technology (AIST), Tsukuba, Japan. From 2001 to 2007, he was at the Research and Development Center, Lion Corporation, Tokyo. His current research interests include polymer science, soft actuator, and nonlinear chemistry. Dr. Hara is a member of the Chemical Society of Japan and the Society of Polymer Science, in Japan. 